

\title{
DISCLAIMER
}

This report was prepared as an account of work sponsored by an agency of the United States Government. Neither the United States Government nor any agency thereof, nor Battelle Memorial Institute, nor any of their employees, makes any warranty, express or implied, or assumes any legal liability or responsibility for the accuracy, completeness, or usefulness of any information, apparatus, product, or process disclosed, or represents that its use would not infringe privately owned rights. Reference herein to any specific commercial product, process, or service by trade name, trademark, manufacturer, or otherwise does not necessarily constitute or imply its endorsement, recommendation, or favoring by the United States Government or any agency thereof, or Battelle Memorial Institute. The views and opinions of authors expressed herein do not necessarily state or reflect those of the United States Government or any agency thereof.

\author{
PACIFIC NORTHWEST NATIONAL LABORATORY \\ operated by \\ BATTELLE \\ for the \\ UNITED STATES DEPARTMENT OF ENERGY \\ under Contract DE-AC05-76RL01830
}

Printed in the United States of America
Available to DOE and DOE contractors from the Office of Scientific and Technical Information,
P.O. Box 62, Oak Ridge, TN 37831-0062;
ph: (865) 576-8401
fax: $(865)$ 576-5728
email: reports@adonis.osti.gov

\begin{abstract}
Available to the public from the National Technical Information Service, U.S. Department of Commerce, 5285 Port Royal Rd., Springfield, VA 22161 ph: (800) 553-6847 fax: $(703) 605-6900$ email: orders@ntis.fedworld.gov online ordering: http://www.ntis.gov/ordering.htm
\end{abstract}

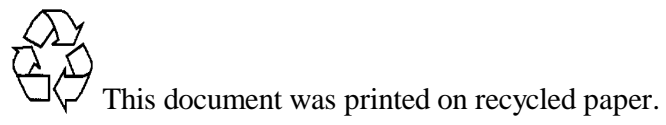


PNNL-17653

\section{Recapitalizing EMSL: Meeting Future Science and Technology Challenges}

AR Felmy

July 2008

Prepared for the U.S. Department of Energy's Office of Biological and Environmental Research under Contract DE-AC05-76RL01830

Pacific Northwest National Laboratory

Richland, Washington 99352 


\section{Contents}

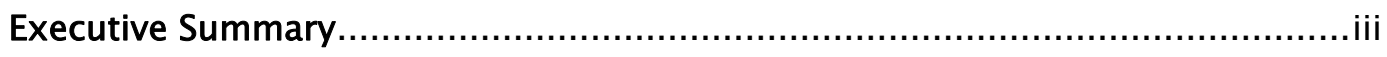

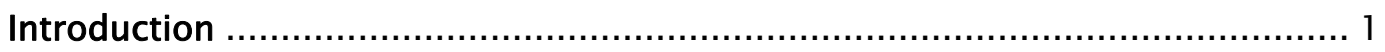

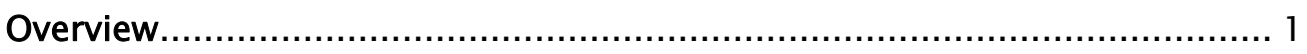

Atmospheric Aerosol Chemistry ....................................................... 9

Biological Interactions and Dynamics ........................................... 11

Geochemistry/Biogeochemistry and Subsurface Science ...................... 15

Science of Interfacial Phenomena ...................................................... 17

Capability Development Driven by Cross-Cutting Molecular Science Themes.... 20

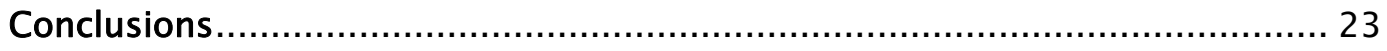




\section{Executive Summary}

EMSL, located in Richland, Washington, is a national scientific user facility operated for the U.S. Department of Energy (DOE) by the Pacific Northwest National Laboratory. The vision that directed the development of EMSL as a problem-solving environment for environmental molecular science has led to significant scientific progress in many areas ranging from subsurface science to atmospheric sciences, and from biochemistry to catalysis. Our scientific staff and users are recognized nationally and internationally for their significant contributions to solving challenging scientific problems. We have explored new scientific frontiers and organized a vibrant and diverse user community in support of our mission as a national scientific user facility that provides integrated experimental and computational resources in the environmental molecular sciences. Users from around the world-from academia

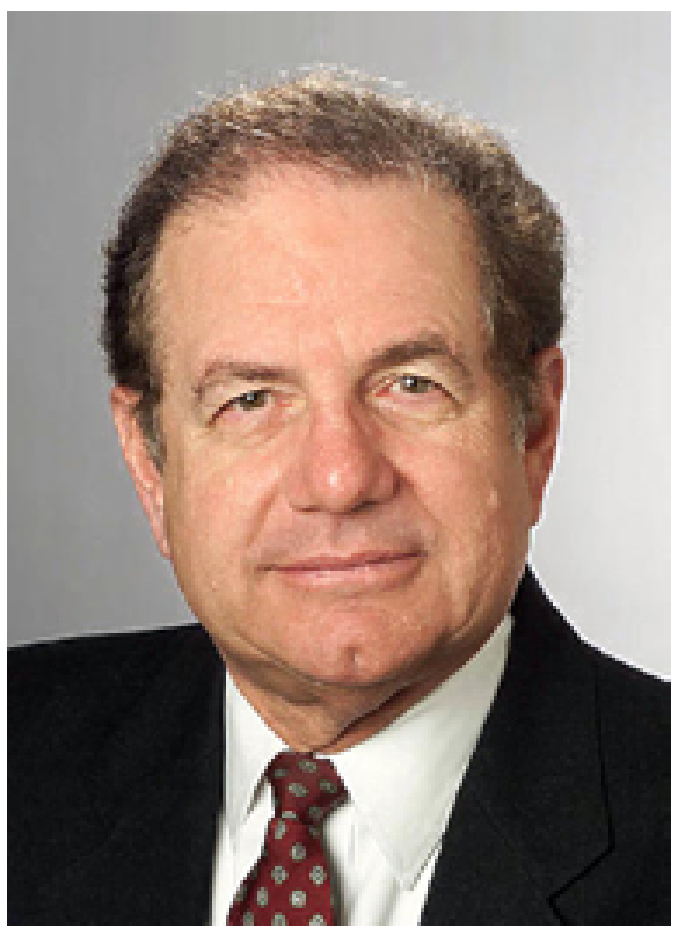
to industry and national laboratories to international research organizations-use the resources of EMSL because of the quality of science that we enable.

In the 21st century, science and technology will be transformed because of our ability to measure, image and control phenomena at the molecular scale combined with equally sophisticated methods for theoretical and computational modeling. The challenge is to We need transformational discoveries, leading to what I call disruptive technologies - technologies that fundamentally change the rules of the game-and that means we need fundamental breakthroughs. integrate this information into appropriate theoretical, experimental, and conceptual frameworks that can link atomic and molecular scales to bulk properties of matter and that can explain its complex and collective behavior. Integration of our understanding of nature requires that we cross many decades of time-from molecular energy transfer times, which may be as short as femtoseconds, to mineral dissolution kinetics that may require hundreds of years - as well as many decades of space-from the nano scale to the macro scale. The unprecedented observational power of modern spectroscopies coupled to massively parallel supercomputers will provide the essential tools while interdisciplinary teams of scientists and engineers provide the intellectual leadership for addressing a broad range of critical problems. 
EMSL is poised to implement these advances to provide a unique problem-solving environment for scientists to create solutions to future challenges in the environmental molecular sciences.

This document defines the science drivers for EMSL's four science themes, built upon the historical scientific expertise at EMSL, around which we will develop and deploy future scientific capabilities. These science themes are Atmospheric Aerosol Chemistry, Biological Interactions and Dynamics, Geochemistry/Biogeochemistry and Subsurface Science, and Science of Interfacial Phenomena. EMSL's ability to issue nationals calls for proposals to the scientific community, coupled with scientific leadership for the conduct of integrated, multi-technique and multi-disciplinary research, a strong dynamic user community, and leading-edge capabilities, will enable EMSL to assist users with conducting innovative research in new ways and establish an enduring and focused national user program to address the energy and environmental science challenges faced by DOE and the nation. Analysis of the science drivers and technologies for each individual science theme has led to the identification of six important cross-cutting development needs that will enable innovative research in multiple areas:

1. Moving from Statics to Dynamics in Native Environments

2. Characterization of Surfaces and Interfaces with Unprecedented Resolution

3. Design and Synthesis of Increasingly Complex Materials

4. Predicting Biological Functions from Molecular and Chemical Data

5. Rapidly Linking Theory with Experiment

6. Bridging Scales: Bridging the Gap between Molecular and Continuum Understanding.

This document also describes the future scientific challenges in each of EMSL's four science themes along with the technology advancements required to push the science forward. It was developed based on a series of planning activities that have taken place over the past three years. Most significant among these activities were five EMSL-sponsored workshops related to developing new capabilities for the user facility:

- “The Environmental Molecular Sciences Laboratory Radiological NMR Spectroscopy Workshop," was held May 1-2, 2006, and included preeminent scientists from six countries who were brought together to discuss the possibilities and value of enhancing radiological capabilities at EMSL, with an emphasis on nuclear magnetic resonance and electron paramagnetic resonance capabilities.

- "The Development of New User Research Capabilities in Environmental Molecular Science" meeting was held August 1-2, 2006, and included 104 expert scientists-many representing EMSL users-from 40 institutions, including 24 universities and 5 national laboratories. Discussions during this meeting centered around future science and technology challenges and the capabilities that these scientists would need in the future to further their research.

- "The NWChem Meeting on Science-Driven Petascale Computing and Capability Development" meeting was held January 25-26, 2007, and included 65 scientists from 22 universities and 
5 national laboratories. Discussions during this meeting centered around status and future direction of NWChem-EMSL's premiere computational chemistry software, how to contribute to the growth of NWChem related to its scientific capability and computational performance by 2022, and parallel implementation and natural linear scaling of coupled-cluster theory of large molecule applications.

- "Science Challenges and Design Concepts for the Next-Generation High-Performance FTICR (Fourier-transform ion cyclotron resonance) Mass Spectrometer" workshop was held January 16-17, 2008, at the National High Magnetic Field Laboratory (NHMFL) in Tallahassee, Florida. The workshop was jointly sponsored by NHMFL and EMSL, and was attended by 32 experts representing the United States, Germany, Korea, Russia, and Sweden. Presentations and discussion sessions focused on science needs for enhanced FT-ICR mass spectrometry capability and identification of technology and instrumentation impediments in reaching the next generation of FT-ICR mass spectrometry performance.

- "The Systems Microbiology and Extremophile Research Facility" workshop was held March 26,2008 , and was attended by 30 scientists, including prominent microbiologists and systems biologists from around the nation. The meeting was held to identify those capabilities needed to build a systems-level understanding of microbes and microbial communities, especially those that can withstand extreme environments. Unique capabilities in cell growth and analysis were identified as well as important roles that EMSL can play in driving progress in the emerging field of systems microbiology.

Additionally, ongoing discussions with EMSL's Scientific and User Advisory committees have provided valuable input and perspective for this document. Finally, feedback from the EMSL User Advisory Committee, as well as feedback from the 2004 Committee of Visitors review of the programs managed by the Environmental Remediation Sciences Division within the DOE Office of Biological and Environmental Research and a 2005 DOE Biological and Environmental Research Advisory Committee review of EMSL's user program, have identified a strong need for maintaining EMSL capabilities at the scientific forefront.

The vision outlined in this document is not an extrapolation of ongoing science at EMSL, but a visionary look towards the new areas of opportunity where advances in environmental molecular sciences and the tools that enable research can have a significant impact. Current and developing technologies can be combined in new ways to create a new generation of research tools, and this vision provides a roadmap for the next decade of scientific capabilities within EMSL that can collectively position EMSL to meet challenges in the environmental molecular sciences. 


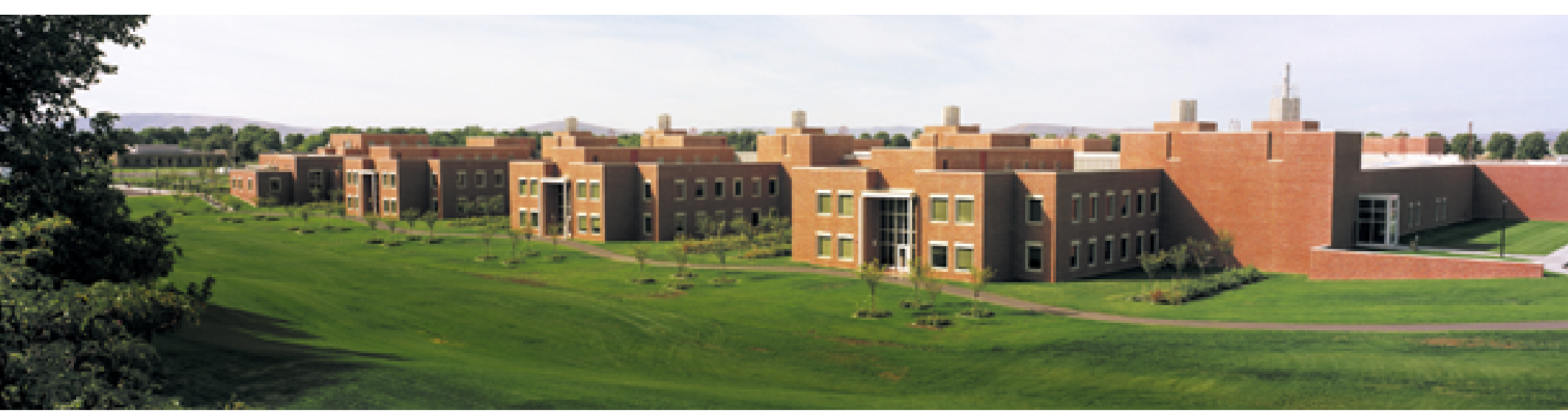

Figure 1. EMSL is a national scientific user facility operated by PNNL on behalf of the Department of Energy.

\section{Introduction}

\section{Overview}

EMSL (Figure 1) is a national scientific user facility funded by the U.S. Department of Energy's (DOE's) Office of Biological and Environmental Research (BER) and operated by the Pacific Northwest National Laboratory (PNNL) in Richland, Washington. Opened to the scientific user community on October 1, 1997, EMSL is a unique facility that integrates state-of-the-art experimental tools and techniques and high-performance computing capabilities with an expert staff to address the complex energy and environmental challenges facing DOE and the nation. Our success reflects our commitment to four "signature" characteristics of EMSL as a national scientific user facility:

1. Developing transformational tools that advance scientific frontiers in EMSL's molecular science themes

2. Adding value by integrating science across disciplines and scientific capabilities

3. Empowering a distinguished community of users that advocates and in invested in the longterm success of EMSL

4. Management excellence that propels EMSL into a long-term, upward spiral of accomplishment and performance.

EMSL capabilities and user projects support important research of EMSL's sponsor, BER, such as the GTL:Genomics Program, and research activities supported by other parts of DOE as well as well as other agencies, including the National Science Foundation and National Institutes of Health. BER supports world-class research in the biological, physical, and environmental sciences to provide innovative solutions to the nation's environmental and energy challenges. Environmental research funded by BER focuses on improving the understanding and reliable prediction of climate change and providing science-based solutions for environmental remediation. Programs in the biological sciences support research in genomics and systems biology, where the goal is to understand how living organisms work as well as how they interact with and react to their environment. This research will enable the development of biological 
solutions to produce clean energy, clean up metals and radionuclides in the environment, and reduce carbon dioxide in the atmosphere. BER's Environmental Remediation Sciences Division, the sponsor of EMSL, has a core mission to advance the fundamental science that will lead to solutions for complex environmental problems such as remediation at DOE waste sites.

Research at EMSL is focused on understanding the physical, chemical, and biological mechanisms that govern environmental processes, starting from the molecular scale and building towards the mesoscale. Specifically, EMSL has established a distinctive signature with its four science themes: Atmospheric Aerosol Chemistry, Biological Interactions and Dynamics, Geochemistry/ Biogeochemistry and Subsurface Science, and Science of Interfacial Phenomena. EMSL issues national calls for proposals to exploit its transformational and state-of-the-art capabilities, thus promoting the development of a strong and dynamic scientific user community engaged in solving DOE-relevant problems. It also provides scientific leadership in the development and implementation of leading-edge capabilities, which is intended to establish an enduring infrastructure to address the science challenges faced by DOE and the nation.

\section{Users Diversity}

EMSL has supported the research programs of an average of approximately 1000 users for each of the last 2 years (Figure

2). Fifty-nine EMSL users are distinguished scientists, as determined by cited publications, endowed chairs, national academy membership, or Nobel Prizes. A distribution of users by affiliation shows that 50 percent of the scientists come from universities, whereas 12 percent of the users are from Federalsponsored laboratories, with the remainder from EMSL, PNNL and other institutions.

\section{Scientific Impact}

With more than 2500 user and staff publications in peerreviewed scientific journals during EMSL's first decade of operation, EMSL is enabling users to make a significant contribution to the scientific understanding of the nation's complex energy and environmental problems. In Fiscal Year 2007, EMSL staff and user research appeared in more than

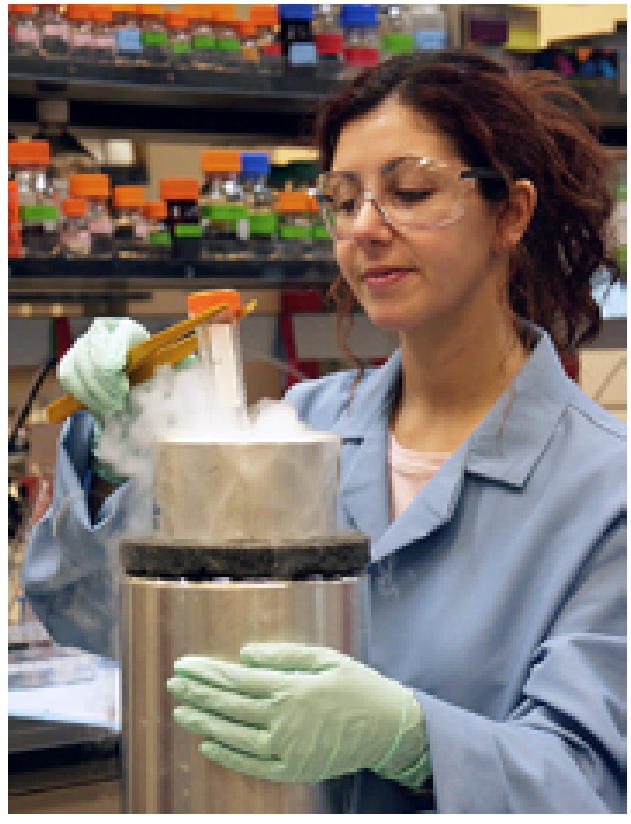

Figure 2. EMSL has supported the research of approximately 1000 users for each of the last 2 years. Half are university users, while the remainder comes from Federal-sponsored laboratories, industry, and national labs. 300 articles including many in prestigious journals such as Science, Nature, Proceedings of the National Academy of Science, and Journal of the American Chemical Society.

EMSL facilitates scientific advances by integrating unique and leading-edge experimental tools with high-performance computing optimized for research areas in EMSL's science themes. Because of the expertise of EMSL's scientific staff, users are enabled to investigate the complex physical, chemical and 


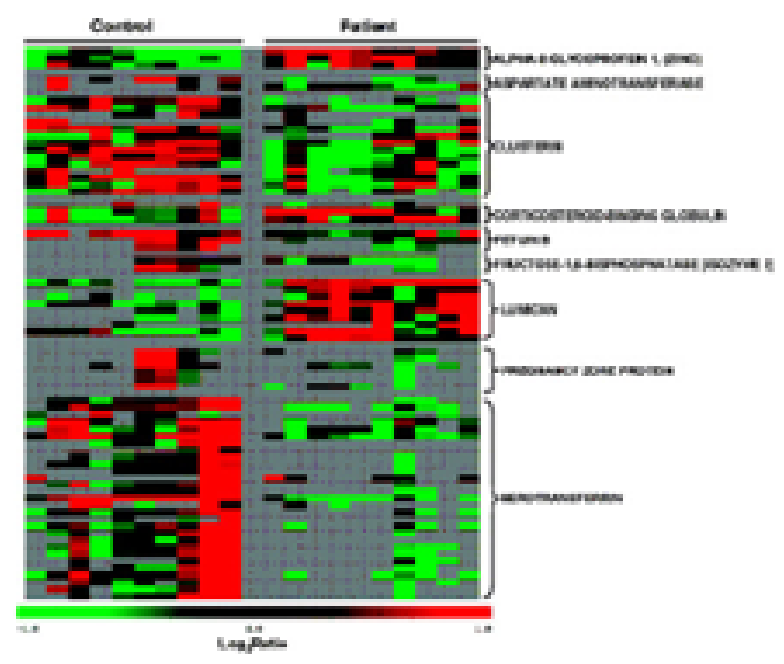

Figure 3. Using EMSL proteomics capabilities, scientific users from the Centers for Disease Control and PNNL discovered five proteins that could help predict type 1 diabetes with higher sensitivity and specificity than current methods. Research results were published in the Journal of Proteome Research [7(2):698-707]. biological nature of many of the nation's energy and environmental issues. Some of the worldleading capabilities that EMSL has developed over the last decade include the following:

EMSL houses a world-leading facility for high-throughput proteomics measurement and characterization (see Figure 3 for a highlight). Developed with EMSL and programmatic resources, the capability is based on customized high-performance liquid chromatography coupled with high-resolution mass spectrometry [Fouriertransform ion cyclotron resonance (FT-ICR), timeof-flight, and Orbitrap] instrumentation capability, and complemented with comprehensive data interpretation and informatics software and tools.

A current throughput of approximately 100 proteome samples per day can be accommodated. A current database of nearly 1 million peptides enables comprehensive eukaryotic and prokaryotic protein identification. A particularly strong capability in microbial proteomics has been developed and is being applied to environmental remediation, carbon sequestration, and energy development problems.

EMSL was the first national scientific user facility to recognize the power of combining highperformance computational capabilities with the development of specialized software that can take advantage of these new resources to enable major scientific progress in a specific area. EMSL scientists continue to lead the development of DOE's premier computational chemistry tool (NWChem) to ensure that researchers can solve evolving scientific questions in areas such as catalysis, solar energy, hydrogen economy, environmental remediation, and biology, while including the complex dynamical behavior of nature in realistic environments. NWChem is the world's leading high performance computational chemistry software suite that provides researchers with the unique capability to understand complex challenging scientific problems at the molecular level by coupling the power of advanced computational chemistry techniques with existing and rapidly evolving high-performance, massively parallel computing
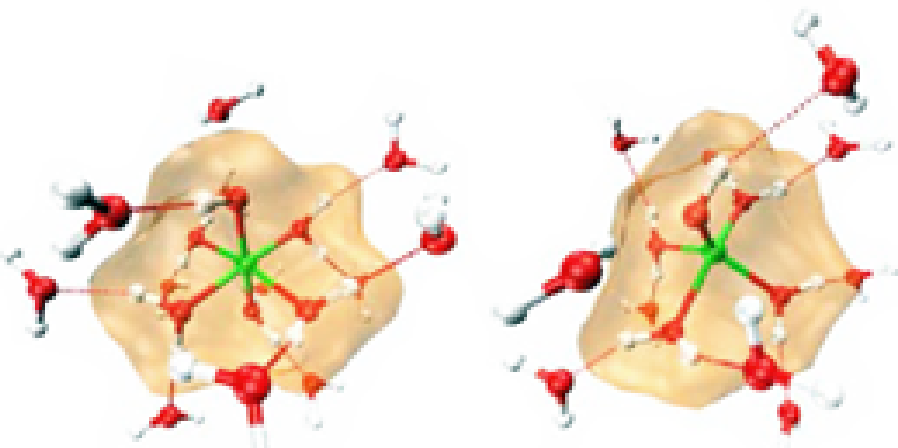

Figure 4. EMSL's high-performance, 11.8-terfaflop supercomputer combined with its premiere computational chemistry software, NWChem, allowed a research team from the University of California, Davis; University of Calgary; PNNL; and State University of New York to determine the structure of a five-coordinate aluminum ion in solution. The results were published in Science [308(5727):1450-1453]. 
systems. As part of the Molecular Science Software Suite (MS ${ }^{3}$, NWChem has been recognized with national awards [R\&D 100 and Federal Laboratory Consortium (FLC) for excellence in technology transfer] and has been downloaded by more than 1800 computing sites worldwide. NWChem running on EMSL's supercomputing hardware enabled the reliable modeling of the thermodynamics cycle of hydrogen release and uptake for chemical boron-nitrogen hydrogen storage compounds, a critical part in the development of environmentally friendly hydrogenbased fuel cells as a power source. It was also used to confirm the discovery of a five-coordinate $\mathrm{Al}\left(\mathrm{H}_{2} \mathrm{O}\right)_{4} \mathrm{OH}^{2+}$ ion as the predominant form in biochemically and geochemically relevant solution environments such as natural bodies of water (see Figure 4 for a highlight).

\section{EMSL, along with the National}

\section{High Magnetic Field Laboratory}

(NHMFL) at Florida State

University, have been the world's

leaders in enabling the benefits

of high field nuclear magnetic resonance (NMR) to be applied to a wide range of complex

systems. EMSL houses a suite of cutting-edge magnetic resonance spectrometers, including a powerful 900-MHz nuclear magnetic resonance system and the world's only NMR spectrometer and laboratory that are dedicated to the study of solid-phase radiological samples. This unique suite of

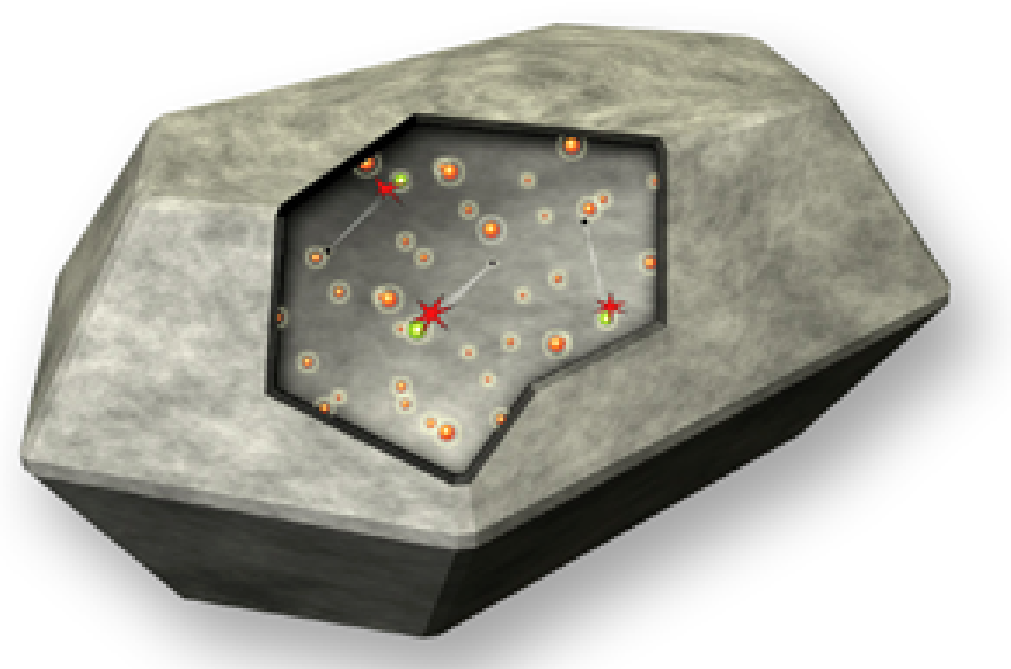

Figure 5. An improved understanding of how alpha particles decay waste forms is leading to enhanced predictive models that support informed decisions on long-term storage of radioactive isotopes. Results of this research, led by a scientific user from the Cambridge University using EMSL's radiological NMR capabilities, were published in Nature [445(7124):190-194]. instruments has contributed broadly to the characterization of biomolecules, surface chemistry, and catalysis research. Special emphasis has been placed on the utilization of the high-field capabilities to determine hard-to-examine nuclei such as isotopes of alkaline earth elements strontium and calcium. The radionuclide investigations contributed to an improved fundamental understanding of the long-term stability of materials proposed for waste immobilization and this is leading to improved predictive models that support informed decisions on long-term storage of radioactive isotopes. As an example (see Figure 5), EMSL users from Cambridge University and the Pacific Northwest National Laboratory published a paper in the January 11, 2007, issue of Nature that describes the impact of alpha emitters on the crystal structure of zircon. The dedicated radionuclide NMR was instrumental in demonstrating unequivocally that radiation effects rapidly degrade the crystal structure of the mineral.

Significant scientific progress has also been achieved by focusing combinations of EMSL's state-of-theart tools and expertise toward important scientific challenges. During the foundation of EMSL, mineral 
and oxide surface chemistry and properties of aqueous systems were identified as two major scientific challenges, requiring both experimental and theoretical development, because of their importance to many environmental topics. More recently, the integrated application of EMSL capabilities has been achieved through the solicitation of "grand challenge" projects in which multiple groups of scientists from around the world come together to solve an important problem.

\section{EMSL is a world leading center for oxide} and mineral surface chemistry research. The ability to synthesize unique oxide films and surfaces along with ability to characterize the physical structure and chemical properties had enabled significant contributions in catalysis, photocatalysis and new-generation oxide-based electronic materials as well playing an important role in advancing the understanding of model biogeochemical systems. Both the research and tool development have been recognized by national awards (2002 Federal Laboratory Consortium Technology Transfer Award and 2004 E.W. Muller Award for Outstanding Research in Surface Science). Recent work (see Figure 6) shows the impact of imaging technologies on our understanding of chemical transformations. Scanning tunneling microscopy measurements of oxygen molecules adsorbing on the surface of rutile $\left[\mathrm{TiO}_{2}(110)\right]$ surface demonstrated that oxygen molecules dissociate only at specific sites

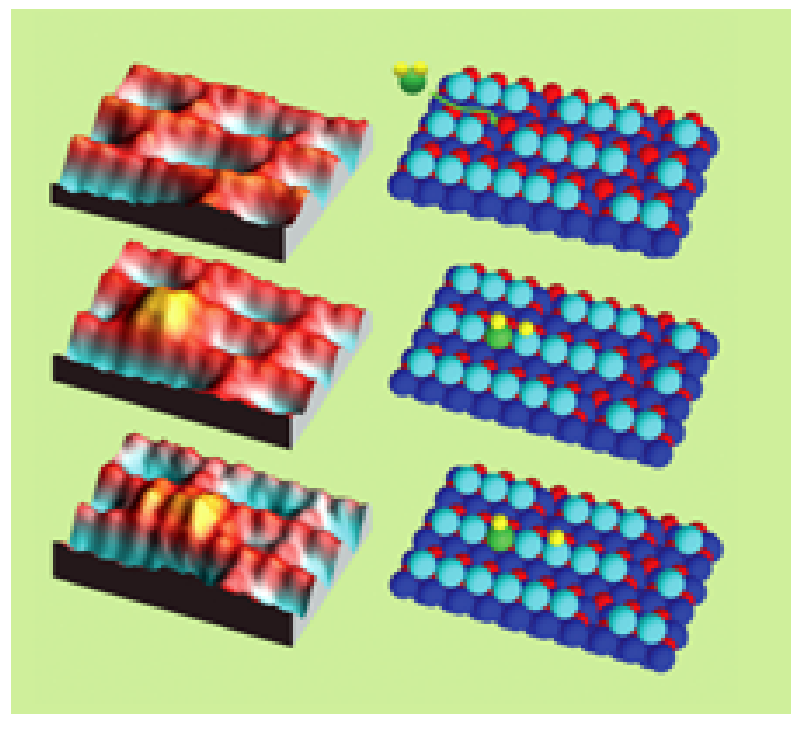

Figure 6. EMSL users from the University of Texas at Austin and PNNL prepared monodispersed supported oxide clusters and made direct observations of the dissociation of water and hydrogen diffusion on a titanium oxide surface, leading to a deeper understanding of model catalytic systems. Through this understanding, scientists may be able to develop new catalytic processes, more efficient solar cells, corrosion-resistant materials, and technologies that split water to generate hydrogen gas. The research was published in Journal of Physical Chemistry B (110:21840-21845)

(bridging oxygen vacancies) with one $\mathrm{O}$ atom healing a vacancy and other $\mathrm{O}$ atom bonding at the neighboring Ti site. This observation is of particular interest not only because it is a unique observation of site specific adsorption, but because $\mathrm{TiO}_{2}$ is an important catalyst substrate and the base material for photocatalytic destruction of contaminant molecules and the possible production of hydrogen.

EMSL was the first National

Scientific User facility to embrace the concept of "Grand Challenge" science. The potential for focused or aligned research efforts to rapidly drive scientific advances was recognized with the opening of EMSL in 1997, when the new
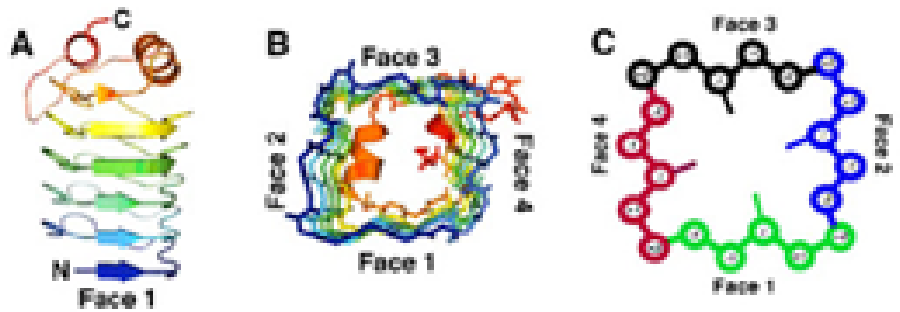

Figure 7. As part of EMSL's Membrane Biology Grand Challenge, the crystal structure was determined for $\mathrm{Rfr} 32$, which is one of 35 pentapeptide repeat proteins (PRPs) in the diurnal cyanobacterium Cyanothece. Results were published in Protein Science [15(11):2579-2595]. Such research is providing insight into nature's methods for carbon sequestration and nitrogen fixation. 
high-performance computational facility began the allocation of computational resources under a "grand challenge" model. These grand challenge computational projects brought together researchers with diverse backgrounds to conduct simulations that could not be performed without access to high-performance computational facilities and software appropriate for use on such massively parallel machines. The emphasis on promoting grand challenge science continued with the implementation of two EMSL scientific grand challenges starting in 2005 one in biogeochemistry and subsurface science focused on electron transfer processes at the microbe mineral interface and one in the area of biological sciences focused on the membrane biology of prokaryotic systems (see Figures 7 and 8).

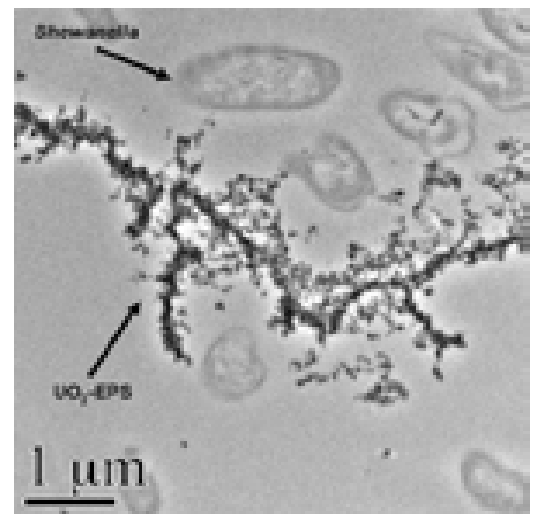

Figure 8. Research under EMSL's Biogeochemistry Grand Challenge to investigate the mechanisms of bacterial extracellular electron transfer to metals and radionuclides revealed that c-type cytochromes-novel redox proteins located on the cell surface-facilitate electron transfer of contaminants such as uranium. The result is the formation of the mineral uraninite, which is relatively immobile in the environment. The research was featured in PLoS Biology [4(8):1324-1333].

\section{EMSL is a world leader in unraveling the complex} interactions that occur in aqueous systems. Water occurs nearly ubiquitously in environmental systems and unraveling the molecular-level interactions of ions and contaminants in water (both in solution, at surfaces, and in the atmosphere) is of fundamental importance in environmental molecular science. This unprecedented understanding of aqueous environments begins with unique capabilities for studying ion-water clusters in the gas phase. Combining electrospray ionization with laser detachment anion beam photoelectron spectroscopy has enabled the production and spectroscopic characterization of multiplycharged, water solvated, gas-phase cluster ions ubiquitous in aqueous solution but heretofore unattainable in the gas phase. Using state of

the art molecular-, ion-, and electron-beam instrumentation in EMSL, EMSL scientific users have pioneered the use of nanoscale amorphous solid water (ASW) films to study ion solvation in solution and ice nucleation and crystallization in the upper atmosphere. Water interactions at solid and mineral surfaces are also fundamental to unraveling the chemical behavior of environmental systems and this has been a focus of EMSL research from the beginning. As an example, EMSL researcher MA Henderson published a comprehensive review of the work in this area which addressed such areas as how water is adsorbed, what are the chemical and electrostatic forces that constitute the adsorbed layer, and how do coadsorbates influence

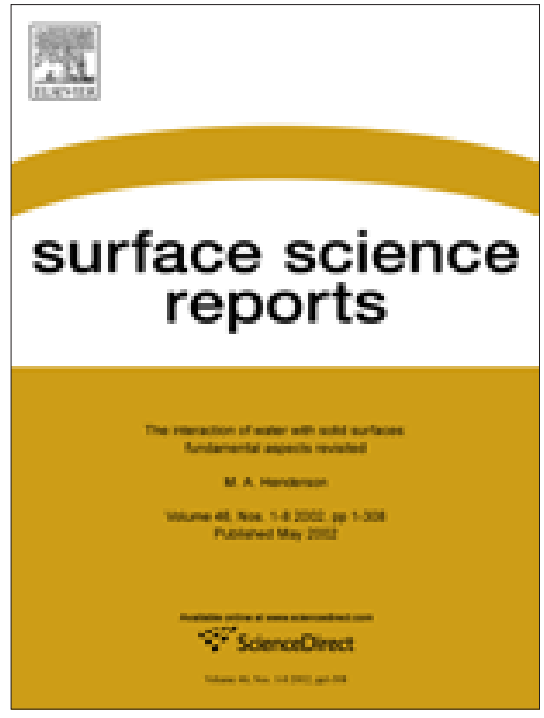

Figure 9. EMSL user Mike Henderson's (PNNL) seminal work reviewing interactions of water with solid surfaces was the subject of a review he published in a 2002 issue of Surface Science Reports. In his review, experimental studies published since a major review published by Thiel and Madey in 1987 were assimilated with those covered by Thiel and Madey to provide a current picture of the fundamental interactions of water with solid surfaces. 
these properties of water (Figure 9). Equally important are advances in the theory, modeling, and simulation of the properties of aqueous environments enabled by the world-class EMSL hardware and software computational resources. Specifically, systematic approaches have been developed to assess the accuracy of various levels of electronic structure theory and they have been applied to accurately evaluate several solute-solvent and solvent-solvent intermolecular interactions. This approach has paved the way for the development of a new generation of interaction potentials for water-water, ion-water and water-solute systems.

\section{Scientific Drivers for New Capabilities and their Impact to DOE}

Much of the research at EMSL is focused on finding solutions to environmental and energy problems important to DOE through a fundamental understanding of underlying physical, chemical, and biological processes. Specifically, EMSL is committed to achieving significant progress as well as scientific discoveries in its four sciences focus areas.

EMSL's atmospheric aerosol science theme originates from the impact of aerosols on global climate change. Biological interactions and dynamics research emphasizes the development of a functional understanding of microbes that will enable optimization and manipulation of microbial processes such as $\mathrm{CO}_{2}$ sequestration. Geochemistry/biogeochemistry and subsurface science theme advances a core strength developed in the past decade of singular importance to the Department of Energy cleanup of legacy waste by improving our ability to predict and modify pollutant transport or transformation in the subsurface. The science of interfacial phenomena theme focuses on developing an understanding of molecular structure-function relationships at the atomic level that will allow precise control of interfacial activity and selectivity needed to optimize such processes as catalytic activity and longevity.

With the goal of advancing these four science themes, the scientific direction and resulting general scientific resource requirements were identified from input obtained from scientific leaders associated with the EMSL science themes, EMSL's User and Science Advisory committees, as well as at technical workshops held at EMSL in 2006-2008. These workshops were:

- "The Environmental Molecular Sciences Laboratory Radiological NMR Spectroscopy Workshop," held May 1-2, 2006. This workshop included preeminent scientists from six countries who were brought together to discuss the possibilities and value of enhancing radiological capabilities at EMSL, with an emphasis on nuclear magnetic resonance and electron paramagnetic resonance capabilities.

- "The Development of New User Research Capabilities in Environmental Molecular Science" meeting, held August 1-2, 2006 (Figure 10). This meeting included 104 expert scientistsmany representing EMSL users-from 40 institutions, including 24 universities and 5 national laboratories. Discussions during this meeting centered around future science and technology challenges and the capabilities that these scientists would need in the future to further their research. 
- "The NWChem Meeting on Science-Driven Petascale Computing and Capability Development" meeting, held January 25-26, 2007. This meeting included 65 scientists from 22 universities and 5 national laboratories. Discussions during this meeting centered around status and future direction of NWChem-EMSL's premiere computational chemistry software, how to contribute to the growth of NWChem related to its scientific capability and computational performance by 2022, and parallel implementation and natural linear scaling of coupled-cluster theory of large molecule applications.

- "Science Challenges and Design Concepts for the Next-Generation High-Performance FT-ICR Mass Spectrometer" workshop was held January 16-17, 2008, at the NHMFL in Tallahassee, Florida. The workshop was jointly sponsored by NHMFL and EMSL, and was attended by 32 experts representing the United States, Germany, Korea, Russia, and Sweden. Presentations and discussion sessions focused on science needs for enhanced FT-ICR mass spectrometry capability and identification of technology and instrumentation impediments in reaching the next generation of FT-ICR mass spectrometer performance. Recommendations to initiate

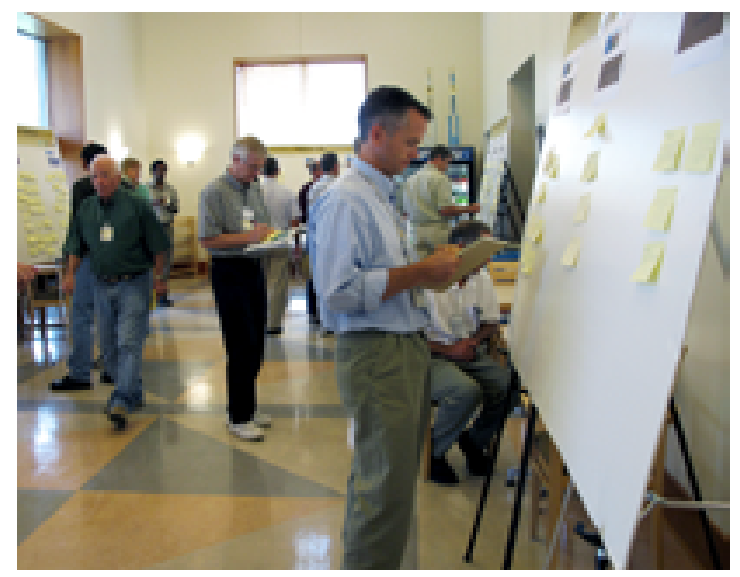

Figure 10. "The Development of New User Research Capabilities in Environmental Molecular Science" meeting was held on August 1-2, 2006, and brought to EMSL 104 expert scientists to discuss future science and technology challenges and required capabilities to further their research. design and construction of a 21-Tesla FT-ICR mass spectrometry system were made (3 to 5 year completion effort) with a view towards application to complex systems science problems (intact-level proteome and protein complex characterization, molecular-level understanding of microbial communities, modern characterization of fossil- and bio-fuels, detailed analysis of organic aerosols, and development of high-resolution imaging capability).

- "The Systems Microbiology and Extremophile Research Facility" workshop was held March 26, 2008, with more than 30 participants, including prominent microbiologists and systems biologists from around the nation. The meeting was held to identify those capabilities needed build a systems-level understanding of microbes and microbial communities, especially those that can withstand extreme environments. Unique capabilities in cell growth and analysis were identified as well as important roles that EMSL can play in driving progress in the emerging field of systems microbiology. The implementation of several pilot projects in systems microbiology was suggested as a way to most rapidly develop the facility, and implementation of a data management system was considered critical to its success.

The announcements for these workshops were broadly distributed to national leaders in all four of the science theme areas in addition to the EMSL user community. Summaries from the workshops were then combined with input from stakeholders, such as BER and the EMSL Science and User Advisory committees. Other sources of input included numerous recapitalization meetings and reviews involving 
EMSL staff and users as well as recent reports from the National Research Council and the Office of Basic Energy Sciences Workshop Reports (www.sc.doe.gov/bes/reports/list.html) including those for geosciences, catalysis and advanced nuclear energy systems. All of these sources form the basis of this document, which describes the science drivers, the associated requirements for enhancing EMSL's scientific resources, and the role that next-generation capabilities will play within 3 to 5 years across the scientific community and for DOE.

The following subsections describe the scientific and technical challenges for each science theme, their impact on DOE environmental missions, and the technology that will be required to greatly advance the research. Although the scientific challenges are different in each area, there are several common themes in technical approach or capability needs: the need to bridge both spatial and temporal scales, methods for dynamical measurements, in situ characterization, and real-time data integration. These cross-cutting molecular science themes are described in the final section.

\section{Atmospheric Aerosol Chemistry}

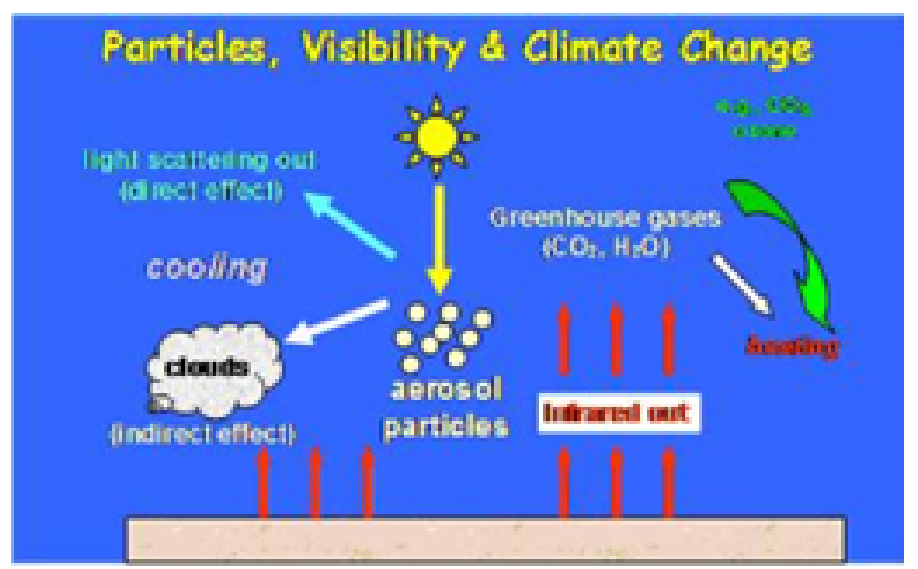

Figure 11. The impacts of aerosol formation on climate change (presentation by Barbara Finlayson-Pitts, University of California, Irvine, at "The Development of New User Research Capabilities in Environmental Molecular Science" meeting, August 1, 2006).
Aerosols (solid and liquid particles suspended in air) influence the Earth's radiation budget through direct radiative forcing by scattering and absorption of incoming solar radiation. They also affect climate indirectly by acting as cloud condensation nuclei and ice nuclei. The extent of direct or indirect radiative forcing by aerosols (see Figure 11) is an area of significant uncertainty in global and regional climate modeling. This uncertainty is due to the variation of mass, composition, and optical properties of tropospheric aerosols on local to regional scales.

Indirect radiative forcing by aerosols is of largest uncertainty. The efficiency of aerosols to act as cloud condensation nuclei and ice nuclei depends largely on their hygroscopic properties. It is generally believed that aerosols composed of highly soluble compounds enhance cloud formation, while those composed of low-solubility constituents inhibit cloud formation. In addition, the chemical composition and physical properties of aerosols evolve during their lifetime as a result of photochemical processing and heterogeneous chemical interactions. Fundamental understanding of aerosol dynamics is needed for accurate atmospheric chemistry and climate modeling. Yet, the role of aerosols, especially organic aerosols, is one of the greatest sources of uncertainty in the interpretation of climate change over the last century and in the modeling of future climate changes. 


\section{Scientific Challenges}

The overall objective of this science theme area as articulated in "The Development of New User Research Capabilities in Environmental Molecular Science" meeting was to understand how physical properties and chemical composition of aerosols affect their interaction with radiation, water, and other gaseous species in the atmosphere and how these characteristics change during the aerosol life cycle. As such, the key science challenges identified related to three processes in the aerosol life cycle: (1) aerosol sources, formation and growth; (2) aerosol transformation; and (3) aerosol impact on cloud formation and eventual deposition from the atmosphere.

Specific science challenges for addressing in the EMSL user program included:

1. Aerosol Sources, Formation, and Growth: Unravel the mechanisms of aerosol particle formation and growth. This includes evaluating the processes leading to new particle formation, measuring the impacts of organics on aerosol particle formation and life cycles, and deciphering the mechanisms of cloud droplet and ice crystal formation and growth in the atmosphere.

2. Aerosol Transformation (Evolution and Aging): Understand the changes that occur inside and on the surfaces of aerosols during their atmospheric lifetime. This includes unraveling the complexities of organic substances (including black carbon) in the atmospheric gas, particle, and droplet phases; their mixing and partitioning on the surface and in the bulk of the aerosols, and their evolution and transformations.

3. Cloud Processes, Scavenging and Deposition: Evaluate how the changing chemical and physical properties of aerosols affect the formation and evolution of cloud droplets and ice crystals and subsequent aerosol deposition. This includes understanding the impact of chemical and physical properties of aerosols on the formation and evolution of cloud droplets and the influence of solution non-ideality on warm cloud formation.

\section{Technical Challenges}

Key technical challenges required to address these science challenges are related to characterization of chemical and physical properties of atmospheric aerosols and their evolution throughout their atmospheric life cycle. These include:

- Development of analytical techniques that operate under ambient conditions (1 atm, $0-100 \% \mathrm{RH}, 230-320 \mathrm{~K}$ ) for analysis of particles both suspended in air and collected on substrates.

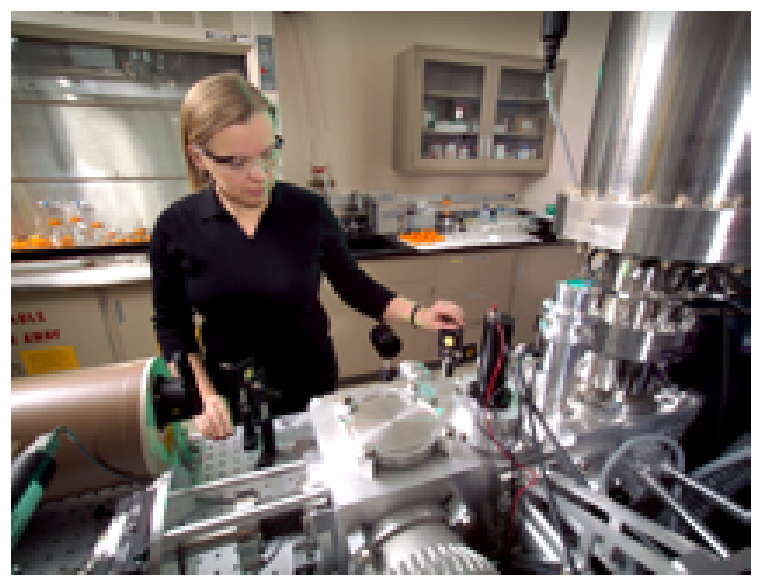

Figure 12. EMSL's field-deployable second-generation single particle laser ablation time-of-flight mass spectrometer-or SPLAT II-is a high-precision and unique instrument that allows researchers to study the fundamental processes that govern the chemistry and physics of aerosol particles on the nano- and micro-scales. 
- Chemical characterization of organic and inorganic aerosols and their transformations (aging, reactivity, condensation) using field-deployable (Figure 12) and laboratory techniques.

- Development of 3-D particle characterization techniques that provide information on heterogeneous properties of individual particles (e.g., particle morphology, bulk and surface composition, optical and hygroscopic properties, particle phases for solids and liquids, aerosol water content) with respect to both inorganics and organics (not just elemental analysis), including speciated molecular information and oxidation states, as a function of position in and coatings on particles.

- Development of sensitive techniques for trace isotope analysis (e.g., using carbon isotopes) of aerosol constituents to distinguish anthropogenic versus natural aerosol sources.

In the area of aerosol formation, the key technical challenges include development of improved insitu chemistry probes for identification of gas-phase precursors and reaction intermediates relevant to formation of secondary organic aerosols; and development of ultra-fast spectroscopic probes of nonequilibrium systems (supercooled water or water vapor) to illuminate the structure and dynamics of nucleation centers for both homogeneous and heterogeneous processes.

Other technical challenges include development of field techniques to ensure collection of representative samples (e.g., in composition, particle size, distribution); development of large-scale data mining and analysis approaches; and web-based techniques for data sharing, storage and access for the scientific user community.

\section{Biological Interactions and Dynamics}

Biological organisms long ago "solved" many problems for which scientists and engineers seek solutions. Microbes in particular offer an astonishingly diverse set of capabilities that can help revolutionize our approach to solving many important DOE problems. For example, photosynthetic organisms can generate hydrogen from light while simultaneously sequestering carbon. Others can produce enzymes that break down cellulose and other biomass to produce liquid fuels. Microbes in water and soil can capture carbon and store it in the earth and ocean depths. Understanding the dynamic interaction between living organisms and the environment is critical to predicting and mitigating the impacts of energy-production-related activities on the environment and human health.

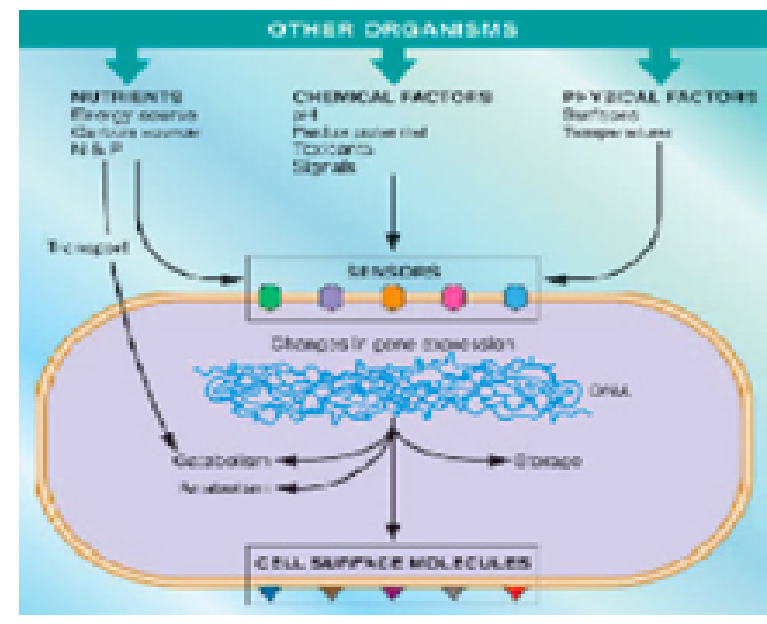

Figure 13. Processes at the microenvironment scale (presentation by Allan Konopka, PNNL, at "The Development of New User Research Capabilities in Environmental Molecular Science" meeting, August 1, 2006).

\section{Scientific Challenge}

Biology is making a transition from a descriptive to a predictive science where biological processes can be both fully understood and productively manipulated at the level of the entire system. This transition 
to systems biology is being driven by the analysis of complex biological processes at scales ranging from the molecular to the whole organism, which requires unprecedented amounts of quantitative data. Both new instruments and conceptual frameworks are needed for enabling the success of this new phase of biological research.

Microorganisms and microbial communities are the focus of DOE research in systems biology because of their potential for solving many critical problems of national importance (Figure 13). In the environment, microorganisms must be able to sense changes in their surroundings and respond appropriately by producing new metabolites, proteins, or other biopolymers. To be able to understand, predict and manipulate these responses, the following key environmental molecular science challenges need to be addressed:

1. What are the molecular-level mechanisms by which microbes sense changes in environmental conditions?

2. What is the architecture of function-specific cellular networks in terms of proteins, multiprotein complexes, metalloproteins, cell surface molecules and metabolites? How are the cells within microbial communities organized into functional networks?

3. What is the composition of molecular complexes that execute specific functions, where are these complexes located and how do they catalyze specific reactions?

\section{Technical Challenges}

The key technical challenges required to address these science challenges occur at different scales of measurement ranging from identifying the microbial community in which the organism exists down to the scale of nanometers in identifying the specific molecular-level structures. For maximum scientific impact, EMSL must provide a range of capabilities to deal with scale dependence. There must also be appropriate data management and analysis systems in place to both organize and understand complex, multi-scale information. The series of technical challenges and their inter-relationships are illustrated in Figure 14.

The first main technical challenge is at the level of the microbial community (see Figure 14, top panel). Capabilities should be developed to grow, identify, and select individual organisms or subsets of organisms from a microbial consortium, especially difficult-to-culture organisms. These facilities should be available within EMSL and allow the ability to vary environmental conditions, observe the microbial community both spatially and temporally, and select specific members of the population for more detailed physiological or molecular-level analysis. This capability is essential to meeting all three scientific challenges but especially the first two listed challenges.

A second main technical challenge is at the cellular and subcellular level (see Figure 14, second and third panels). Capabilities need to be developed to characterize the architecture of living cells, determine the location of specific proteins or metalloproteins, and to isolate individual cellular structures for ex situ detailed molecular level analysis. It was suggested that many of these capabilities be co-located as part 
of an overall Systems Microbiology and Extremophile Research Facility for ease of user access. This capability is critical for the last two listed scientific challenges.

The third main technical challenge is at the level of characterizing specific molecularlevel interactions that occur in biological systems. Capabilities need to be developed to characterize specific posttranslational modifications of proteins, multiprotein complexes and metalloproteins, their 3-D structure, and, where applicable, their catalytic mechanisms. The identification of catalytic mechanisms will require the ability to identify the reactant molecules in real-time along with the associated biological structures.

\section{There is also a need to develop the} computational infrastructure for the next generation of biological studies. Software systems need to be developed that can facilitate the analysis of the large amount of data that can be collected with advanced instrumentation and which support

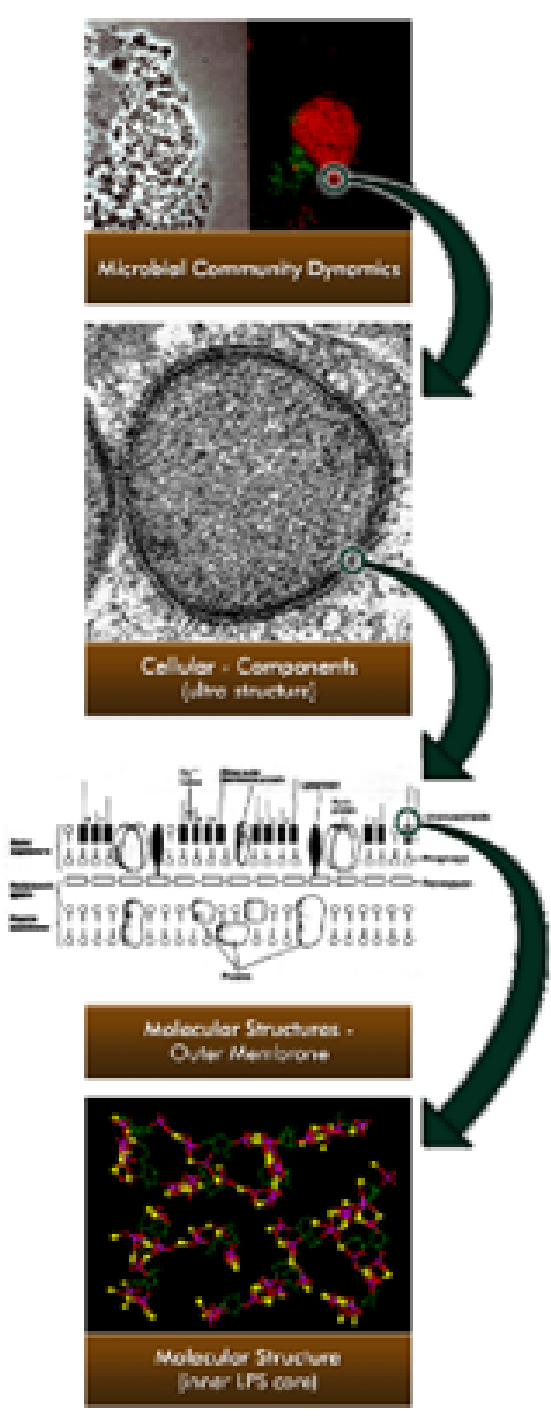

Figure 14

Image on the left is of a microbial consortium. In color on the right is a fluorescent in-situ hybridization image that shows ammonium-oxidizing bacteria in red.

Microbial ultrastructure of a gram-negative bacterium
Diagrammatic representation of the major components in the outer microbial membrane of a gram-negative bacterium.
Molecular dynamic simulation of the inner core of the external lipopolysacchardides in the bacterium Pseudomonas aeruginosa.

predictive modeling and exploit the computer resources at EMSL (Figure 14, bottom panel). This software should also exploit the sequence data that is being generated by other facilities, such as the Joint Genome Institute, and broadly enable the understanding of complex, multicellular systems. Because interaction data are being gathered on multiple spatial and temporal scales, from the atomic to the community level, computational systems that can link these scale is essential. Data linking, integration, analysis, and access are fundamental requirements for enabling the system-level approach to biology that promises to solve important problems in the next several decades.

Several key technical challenges associated with determining multiprotein complexes, posttranslational modifications to proteins, and the catalytic mechanisms of protein function can be addressed by mass spectrometry (Figure 15) or magnetic resonance approaches, which are current strengths within EMSL. Specific recommendations on how these capabilities should be enhanced in the future include:

- Electron transfer and electron capture dissociation mass spectrometry methods for determining posttranslational modifications of proteins. 
- Development of a high-field Fourier transform ion cyclotron resonance mass spectrometer for whole protein analysis and enhanced sensitivity and resolution in analysis of proteome complexity.

- High-field electron paramagnetic resonance (>10 Tesla) capabilities for electron and proton transfer reactions in biological systems.

- New high-sensitivity cryogenically cooled nuclear magnetic resonance probes for examining metalloproteins, multiprotein complexes, and metabolites.

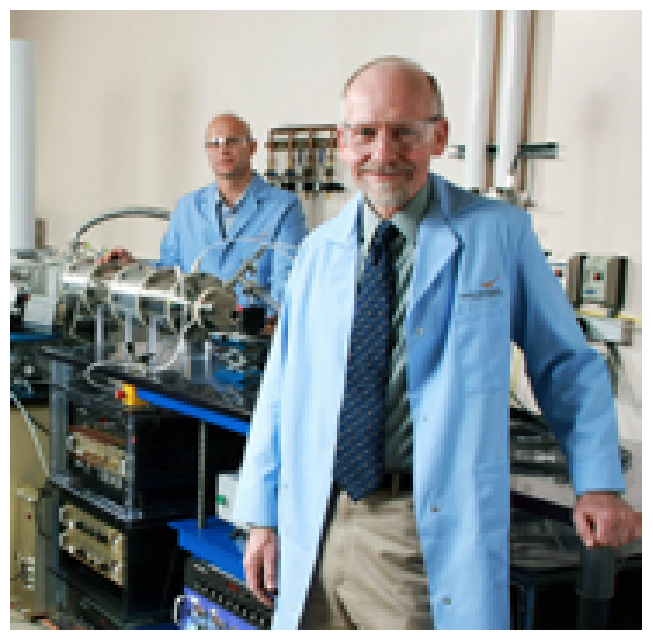

Figure 15. Mass spectrometry is one capability set within EMSL that can be enhanced in the future to continue to address determining multiprotein complexes, posttranslational modifications to proteins, and catalytic mechanisms of protein functions. 


\section{Geochemistry/Biogeochemistry and Subsurface Science}

One of the most challenging and pressing issues confronting DOE and the nation is the safe and costeffective management of environmental pollutants and the remediation of hazardous waste sites. DOE is responsible for managing some 40 million cubic meters of contaminated soils and 1.7 trillion gallons of contaminated ground water. At the Hanford Site in Richland, Washington, alone, millions of gallons of highly radioactive and hazardous wastes in hundreds of underground tanks have leaked, causing extensive contamination of the soil and groundwater. These issues are also national problems. For example, across the United States, thousands of Superfund sites exist with various levels and types of contamination ranging from organics (polychlorinated biphenyls,

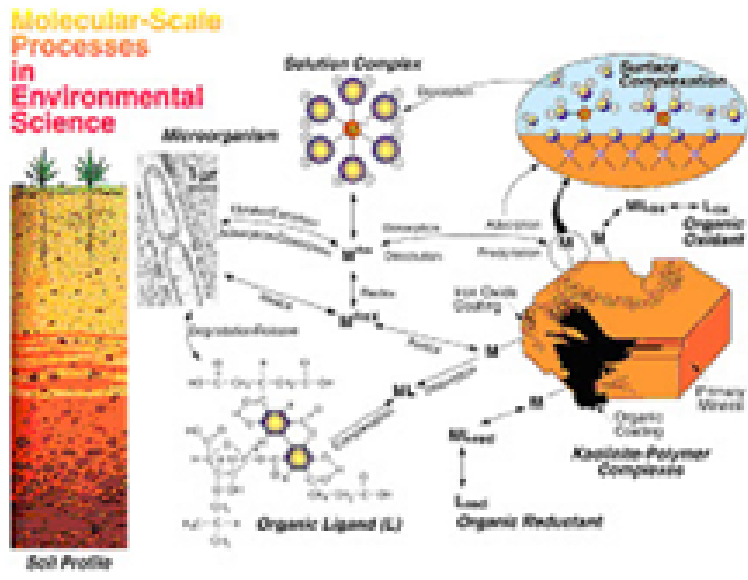

Figure 16. Molecular-level complexity of biogeochemical systems (presentation by Gordon Brown, Stanford University at "The Development of New User Research Capabilities in Environmental Molecular Science" meeting, August 1, 2006). carbon tetrachloride, trichloroethene); heavy metals (mercury, chromium, lead, arsenic); inorganics (phosphates, nitrates) to radionuclides (uranium, technetium, tritium, plutonium, strontium, cesium, americium). The subsurface migration of other byproducts of energy production $\left(\mathrm{CO}_{2}\right)$ also represents one of the most difficult and daunting challenges to the DOE, because the disposal of future generated byproducts $(\mathrm{CO} 2)$ could limit the deployment of new energy technologies.

Molecular-level processes (Figure 16), such as aqueous complexation, adsorption to different mineral phases, or microbial reduction of redox active metals, often control the transport and fate of contaminants in the environment. These processes occur in complicated subsurface environments that are chemically and physically heterogeneous. Understanding the structure, chemistry, and nanoscale geometric properties of the mineral/water and microbe/mineral interfaces are therefore key aspects of developing a mechanistic understanding of subsurface reactivity and contaminant transport. As a result, molecular-level studies of interfacial geochemistry and biogeochemical reactions have been an active area of research for more than a decade. Unraveling these phenomena at the molecular level and determining their impact on contaminant migration and transformation in the environment is a key objective of this science theme area.

\section{Scientific Challenges}

With this general understanding of subsurface systems, participants at "The Development of New User Research Capabilities in Environmental Molecular Science" meeting outlined three key scientific challenges in the areas of nanobiogeoscience, system complexity, and bridging spatial and temporal scales. These challenges were identified in the context of where EMSL could have a significant scientific impact on unraveling the mechanisms behind the fate and transport of contaminants and other constituents in subsurface systems. 
1. Nanobiogeoscience: Unraveling the genesis, properties, and effects of nanominerals and nanostructured materials (anthropogenic and natural) in the environment. This scientific challenge includes understanding mechanisms of biomineralization at the molecular level (e.g., bacteria-expressed nanomaterial synthesis capabilities that offer exquisite control on product properties and morphology) and elucidating the structure, unique properties, reactivity, and transport of nanostructured materials in the environment.

2. Natural Complexity and Molecular Resolution: Understanding the dynamics of reactions at complex interfaces with high temporal and spatial resolution. This scientific challenge includes characterizing mineral surface structures including natural topographic heterogeneity and defects in different geochemical environments; determining the impacts of microbes, biofilms, and organics on mineral surface reactivity; and understanding coupled processes (e.g., diffusion/electron transport, surface proximity effects) with molecular resolution (see Figure 17).

3. Bridging Scales: Bridging the gap between molecular and continuum understanding. This scientific challenge includes relating molecular properties to macroscopic properties such as solubility, dissolution, and adsorption; as well as investigating the effects of fluid flow from the microscale (confined spaces and fractures) to the continuum level.

\section{Technical Challenges}

The technology required to address these scientific challenges must deal with the issues of characterizing the complexity of heterogeneous systems, evaluating coupled processes (dynamics), and linking molecular scale and macroscale reactivity (scaling).

- Imaging of complex heterogeneous systems. In working with complex heterogeneous materials the ability to image the system at different scales and selectively isolate fragments or parts of the system for further characterization until ultimately the molecular scale is obtained is of prime importance (see Figure 17).

- In-situ characterization of biomineralization and mineral surface reaction dynamics. Microbial processes and many geochemically driven processes occur across a wide range of time scales. Furthermore, many of these processes are strongly influenced by the chemical and physical conditions of the system in its natural state. Evaluating such processes requires real-time in-situ imaging and spectroscopic capabilities.

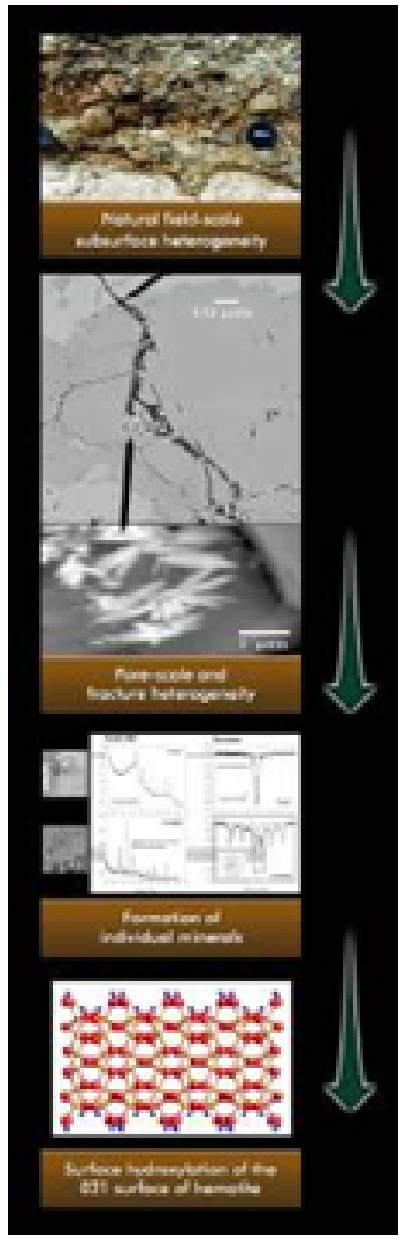

Figure 17. Scale dependence of technical challenges in the area of biogeochemistry and subsurface science (graphic by Andy R. Felmy, EMSL, from "The Development of New User Research Capabilities in Environmental Molecular Science" report, August 2006). 
- Development of techniques for microscale reactive transport studies. EMSL has and will continue to focus on molecular biogeochemistry. However, fluid flow and transport phenomena are also often important in the environment and in laboratory-based systems in the near surface region or in confined spaces. Capabilities need to be developed in microscale reactive transport, adding chemistry on top of microfluidics, to better understand geochemical and biogeochemical reactivity as well as to help bridge the gap between molecular and continuum scales.

In addition to these capabilities, there was strong support for enhanced capabilities to handle radionuclides and for the development of flexible and universal mechanisms for transferring samples between instruments under controlled atmosphere (anoxic) conditions.

\section{Science of Interfacial Phenomena}

Interfaces control many chemical and physical properties of natural and engineered materials critical to environmental- and energy-related research and technology. Tailored or designed surfaces and interfaces are important both as model systems for detailed study of processes that occur on natural heterogeneous materials in the environment and to design materials with new properties for technological use, such as energy production or catalysis. The behaviors of complex heterogeneous materials in the environment can never be fully understood without model systems that allow specific aspects of that complexity to be examined in detail. Likewise, material systems with interfaces optimized with specific properties are essential for the advanced technologies needed for a secure environment and a stable energy future for the nation.

Examples of technologies that rely on improved understanding and control of molecular-level structural, dynamic, and transport properties of interfaces include hydrogen production and storage, chemical sensors and radiation detectors, solid-oxide fuel cell research and development, materials for next-generation nuclear reactors, thin-film solar cells, new generations of selective catalysts, and the development of solid-state lighting. As indicated in Figure 18, sensors, catalysts, photocells and other environmentally important systems usually involve solid-solid, solid-liquid, or solid-gas/vacuum interfaces of a variety of physical structures needed to extract chemical and functional information at the atomic or molecular scale.

This science theme is focused on developing an understanding and gaining control of structurefunction relationships at the atomic level to allow the design of new energy technologies. The science and technological issues that need to be addressed to achieve these goals are complementary to those identified in the atmospheric, geological, and biological areas.

\section{Scientific Challenges}

- Expanding our understanding and ability to rationally design, synthesize, and characterize complex surfaces, films, and interfaces. One focus of this area is to enhance our ability to deal with complex materials, complex structures, and complex environments, some of which are illustrated in Figure 18. Being able to manipulate complex materials and the related interfaces 
will allow the creation of films/materials with designed chemical, electronic, magnetic, and optical properties related to sensor, detector, catalysis, and energy needs. Extension of the level of experimental and theoretical understanding available for metal and semiconductor surfaces to metal oxide, hydroxide, layered silicate and other insulating systems was a scientific challenge undertaken by EMSL at its inception.

- Understanding and controlling the dynamic properties of surfaces and interfaces. We are just beginning to understand the truly dynamic (time and environmental dependent) nature of surface and interface structures that will have major impacts on the nature and presence of defects and reaction sites. These dynamic effects may be more apparent for organic materials or biomaterials, but also apply to what has been commonly viewed as "static" inorganic surfaces and interfaces. This time and environment dependence adds new challenges and opportunities for understanding and controlling structure-function relationships at surfaces and interfaces of many types. These dynamic effects will influence our understanding of chemical reactivity for catalysis, photocatalysis, and bioactive materials. They also have significant implications for processes involving mass and charge transport necessary for detectors, sensors, fuel cells and photovoltaics, as well as offering new approaches to control reaction processes.

Figure 18

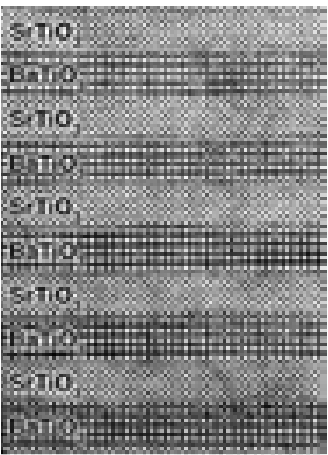

$5 \mathrm{~nm}$

Transmission electron microscopy image of a multi-layer oxide structure grown by molecular beam epitaxy. Such structures are relevant to ferroelectric materials, magnetic components, or sensors and provide one example of buried solidsolid interfaces for which atomic-level order (and deviation from that order) is significant (presentation by Yuri Suzuki, University of California, Berkeley, at "The Development of New User Research Capabilities in Environmental Molecular Science" meeting, August 2, 2006).

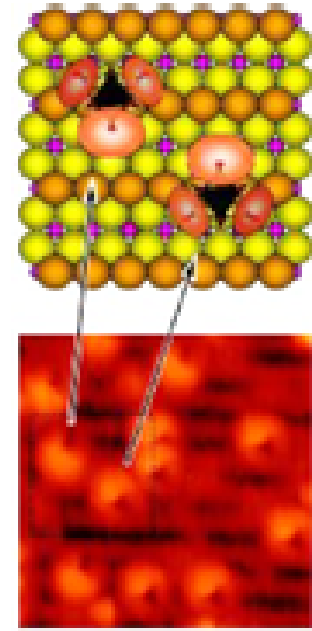

Scanning tunneling microscopy image of catalytically nanometersized active sites on an oxide surface. Specific reactive sites and sometimes defects play a major role in defining the chemical properties of surfaces and interfaces (presentation by J. Mike White, University of Texas at Austin, at "The Development of New User Research Capabilities in Environmental Molecular Science" meeting, August 1, 2006).

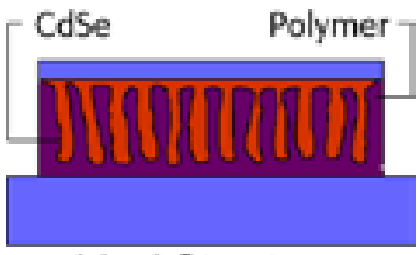

Ideal Structure

Model of future solar cell involving organic and inorganic materials and a complex geometrical structure. The ability to adequately characterize such a complex mixedphase system is an important challenge (presentation by Mowafak Al-Jassim, National Renewable Energy Laboratory, at "The Development of New User Research Capabilities in Environmental Molecular Science" meeting, August 1 2006).

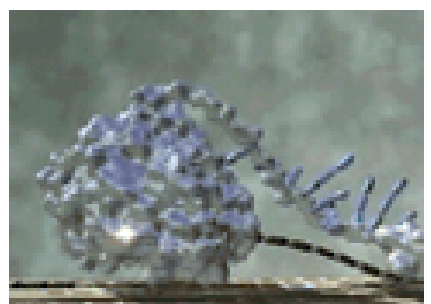

Drawing of endoglucanase on a cellulose surface. Biological surfaces and interfaces are a challenge to fully characterize, but play a major role in biomass conversion (presentation by Mark Davis, National Renewable Energy Laboratory, at "The Development of New User Research

Capabilities in Environmental Molecular Science" meeting, August 1, 2006) 


\section{Technical Challenges}

Several important technical challenges in the areas of material synthesis, capabilities for probing single sites on surfaces, and in situ/operado/real-time capabilities, were identified.

- In-situ/Operando/real-time probes. The ability to measure chemical and structural characteristics of surfaces and nanostructured materials as a function of environment and time is critical for understanding the dynamical and transient behaviors of materials and interfaces relevant for a more advanced understanding of structure-property relationships. Capability developments that facilitate in situ capabilities should be emphasized.

- High-resolution and interfacial analysis tools. Information must be obtained about the structure, composition, and chemical properties of specific (single) sites and small areas. The ability to probe atomic and molecular structure and composition of solid/solid (including organic/inorganic, inorganic/inorganic and organic/organic); solid/liquid; and solid/gas interfaces is critically important and should include chemical imaging to the extent possible. It is increasingly important to combine spectroscopy (physical and chemical information) and microscopy (spatial information). High-resolution tools are also important for engineering natural materials.

- Sample synthesis and preparation capabilities. Important needs in this area include the synthesis of complex (composition and morphology) oxide interfaces, films, and surfaces as well as developing methods to observe and control growth processes in real time, including in solution. The ability to prepare and handle samples to retain desired properties is a special and increasingly important challenge. The integration of precision calorimetric tools with nanostructure synthesis can provide the experimental basis for testing in-detail theoretical model accuracy.

Another important issue specifically noted at "The Development of New User Research Capabilities in Environmental Molecular Science" meeting was the need to develop a new level of verified theory relevant for interfaces and nanostructures. Although fundamental theoretical tools have been developed to the point where they can contribute to our understanding of molecular structures with increasingly large numbers of atoms and complexity, it is still difficult to experimentally validate theoretical predictions regarding nanostructures and interfaces. Although it is possible to verify calculated structures, kinetic and energetics provide a more critical test of accuracy. An important goal is to be able to calculate energetics with the accuracy needed for chemical predictions. 


\section{Capability Development Driven by Cross- Cutting Molecular Science Themes}

An analysis of the major technical challenges faced by each science theme shows that a few cross-cutting advances in molecular science capability would have major impacts on future science for all of the science themes. These capabilities would broadly underpin different science areas from subsurface fate and transport to the development of new energy systems. By focusing future capability development on these cross-cutting molecular science capabilities, it becomes possible to develop an investment strategy that will more broadly impact the different EMSL science challenges. This section highlights these crosscutting molecular science themes and gives specific examples of capabilities that would address them. The cross cutting themes reflect general directions. The detailed development of these capabilities is best determined by a "rolling" capital investment plan that is updated at least once a year to reflect funding and technology advances.

\section{Moving from Statics to Dynamics in Native Environments}

We are just beginning to understand the truly dynamic (time and environmental dependent) nature of surfaces and interfaces that have major impacts on environmental processes. The ability to measure chemical and structural characteristics of surfaces and nanostructured materials in their reactive environment is critical for understanding the time-dependent behaviors of materials and interfaces relevant for a more advanced understanding of structure-property relationships. These dynamic effects will influence our understanding of chemical reactivity (catalysis, photocatalysis, bioactive materials); have significant implications for processes involving mass and charge transport (detectors, sensors, fuel cells, photovoltaics); and may offer new approaches to control reaction processes.

A suite of complementary in situ and real-time methods is needed to collect high-resolution structural and chemical information, such as can be accomplished using high-resolution transmission electron microscopy with environmental cells. There is also a need to define particle morphology and chemical structure by using approaches such as environmental scanning electron microscopy combined with spectroscopy. Finally, there is a strong need to probe molecular chemistry and reaction species in different environments and to delineate molecular structure and trace element composition at solid/liquid interfaces. The former can be accomplished using integrated optical systems, whereas the latter can be accomplished using a custom-designed secondary ion mass spectrometer.

\section{Characterization of Surfaces and Interfaces with Unprecedented Resolution}

The ability to probe atomic and molecular structures and chemical composition, and image solid/ solid (including organic/inorganic, inorganic/inorganic and organic/organic); solid/liquid; and solid/ gas interfaces at the nanometer scale is critically important to understanding the reactive properties of both natural and synthesized materials. It is therefore increasingly important to combine spectroscopy (physical and chemical information) and microscopy (spatial information) for three-dimensional analysis 
of structures, including buried interfaces. The differing nature of these interfaces and the wide range of molecular, structural, and elemental information that is needed will necessitate a complementary set of tools. The required suite of tools must collect information on atomic composition (including trace elements and isotope ratios) at high resolution, which can be accomplished using nanoSIMS capability. The needed high-resolution structural and chemical information can be obtained by a transmission electron microscope, whereas probing surface chemical reactions at single sites will require a custom surface chemistry and scanning probe microscopy system. Advanced instrumentation is also needed to obtain structural and chemical analysis of intact buried interfaces and advanced three-dimensional structural and chemical analysis.

\section{Design and Synthesis of Increasingly Complex Materials}

Tailored or designed surfaces and interfaces are important both as model systems for detailed study of processes that occur on natural heterogeneous materials in the environment and to design materials with new properties for technological use, such as energy production or catalysis. It is likely that the behaviors of complex heterogeneous materials in the environment can never be fully understood without model systems that enable behaviors of specific aspects of that complexity to be examined in detail. Likewise, material systems with interfaces designed (tailored) and optimized to have specific properties are essential to advanced technologies needed to maintain a secure environment and obtain a stable energy future for the nation. To address these science issues, EMSL must offer a range of unique and state-of-the-art capabilities to advance understanding of complex interfaces and surfaces. New capabilities important in this area include oxygen plasma-assisted molecular beam epitaxy and high-throughput materials production and analysis. These synthesis capabilities will be designed to be linked to sample preparation and analysis capabilities to enable sample handling in controlled environments and well-defined conditions.

\section{Predicting Biological Functions from Molecular and Chemical Data}

Biology is making a transition from a qualitative science to a quantitative, and ultimately predictive, science. This transition is driven by the large amounts of molecular data that can be obtained by new high-throughput technologies and by the increasing power of computational analysis tools. The ability to link the physiological behavior of organisms to their molecular properties is crucial for developing a predictive understanding of organisms. This will be the foundation for reengineering organisms to meet the technological challenges of clean and sustainable energy as well as to minimize the environmental impact of current energy technologies.

Growing and characterizing living cells under unique and challenging growth conditions require an integrated suite of instruments with automated sampling and computer-controlled monitoring capabilities. Understanding the complexity of multicellular systems will also require equipment capable of visualizing processes and molecular structures within living cells. Quantitatively characterizing complex microorganisms and their communities at the molecular level will require a new generation of mass spectrometers of unprecedented speed and resolution. Analyzing multiprotein complexes, posttranslational modifications to proteins, and the catalytic mechanisms of protein function will also require improved mass spectrometry and magnetic resonance instrumentation within EMSL. 
The interaction of microbes with surfaces is essential for understanding how they function in communities and interact with the environment. Thus, EMSL capabilities in analyzing interfaces and molecular-level structures can be productively applied to understanding biological systems. However, integrating and making sense of all of these different types of data require both conceptual and mathematical frameworks that can link the mechanistic details of biological organisms to their higher-level functions. It also requires the storage of the relevant data in a central repository and the creation of a computational framework for modeling key biological processes. An integrated data management system that provides users with access to all of the data generated at EMSL is essential for enabling systems biology research.

\section{Rapidly Linking Theory with Experiment}

Users often have only a limited time to conduct their research while at EMSL. The analysis of their experimental data using the most sophisticated theoretical models while they are still at EMSL enables experiments to be altered to test new hypotheses. Rapid linking of experiment and theory would provide the opportunity for a qualitative change in the way experiments are designed, conducted, and analyzed. Several experimental methods have been identified for which near real-time analysis can significantly advance the quality and relevance of the data being collected. These include electron microscopy and spectroscopy, nuclear magnetic resonance, and other techniques for which rapid analysis could identify the structure and composition of materials as they are prepared and analyzed. Implementing a rapid analysis strategy would require the ability to transparently transfer, store, integrate, process, and mine data from a variety of sources and would place EMSL at the forefront in this capability area.

\section{Bridging Scales: Bridging the Gap between Molecular and Continuum Understanding}

This scientific challenge is to relate molecular properties of materials to macroscopic reactivity. As such, it crosses over all four of the EMSL science themes, from unraveling the role of molecular processes in mineral dissolution in subsurface science, to developing better catalysts in the area of interfacial science, to understanding biological function from an analysis of their pattern of gene expression and molecular composition, and to developing improved predictive models of climate change based upon the molecular behavior of aerosol particles. Advancements in computational science is especially important to drive progress in this area since it can provide the theoretical basis and technical capability to quantitatively link information from different spatial and temporal scales. 


\section{Conclusions}

These cross-cutting molecular science themes provide the basis of a vision that will be used to guide the capability development in EMSL over the next decade. Implementation of this vision will require creating unique state-of-the-art capabilities in several areas as well as linking multiple, previously separate tools that collect information at different time or spatial scales. Implementation will also require using the best current technology and incorporating new technology developed both in EMSL and by others. The creation of a suite of integrated analytical tools will provide a totally unique set of capabilities for advancing the environmental molecular sciences and solving scientific problems important to both DOE and the nation. Meeting the objectives of these themes, as well as the overall multi-discipline mission of EMSL, requires integrating instruments, modeling, data collection and analysis, and facility operation in unprecedented ways. 
